\title{
LMI Techniques for Optimization Over Polynomials in Control: A Survey
}

\author{
Graziano Chesi, Senior Member, IEEE
}

\begin{abstract}
Numerous tasks in control systems involve optimization problems over polynomials, and unfortunately these problems are in general nonconvex. In order to cope with this difficulty, linear matrix inequality (LMI) techniques have been introduced because they allow one to obtain bounds to the sought solution by solving convex optimization problems and because the conservatism of these bounds can be decreased in general by suitably increasing the size of the problems. This survey aims to provide the reader with a significant overview of the LMI techniques that are used in control systems for tackling optimization problems over polynomials, describing approaches such as decomposition in sum of squares, Positivstellensatz, theory of moments, Pólya's theorem, and matrix dilation. Moreover, it aims to provide a collection of the essential problems in control systems where these LMI techniques are used, such as stability and performance investigations in nonlinear systems, uncertain systems, time-delay systems, and genetic regulatory networks. It is expected that this survey may be a concise useful reference for all readers.
\end{abstract}

Index Terms-Control system, linear matrix inequality (LMI), optimization, polynomial, positivity.

\section{INTRODUCTION}

$\mathbf{I}$ $\mathrm{T}$ is well-established that the analysis and synthesis of control systems often involve the solution of optimization problems over polynomials, such as determining the minimum of a polynomial under polynomial inequality constraints, or searching for a polynomial satisfying such constraints. Indeed, the basic problem of establishing stability of an equilibrium point of a system via Lyapunov functions consists of determining a positive function such that its temporal derivative along the trajectories of the system is negative outside the equilibrium. Such a problem, and many similar ones investigating other key issues of control systems, are naturally optimization problems over polynomials in the case that the temporal derivative of the state of the system and the candidate Lyapunov function are polynomials. Moreover, appropriate transformations or approximations allow one to consider in this framework also several other cases involving nonpolynomial nonlinear functions. See for instance [1].

Unfortunately, optimization problems over polynomials are in general nonconvex. In fact, in a nonconvex optimization problem there can be local optima in addition to the global

Manuscript received July 14, 2008; revised July 07, 2009; accepted March 15, 2010. First published April 05, 2010; current version published November 03, 2010. This work was supported in part by the Research Grants Council of Hong Kong (HKU711208E). Recommended by Associate Editor P. Parrilo.

The author is with the Department of Electrical and Electronic Engineering, University of Hong Kong, China (e-mail: chesi@eee.hku.hk).

Digital Object Identifier 10.1109/TAC.2010.2046926 one, and hence it is difficult to determine the global optimum via standard numerical methods which are able to establish optimality of a solution only locally. In order to cope with this difficulty, researchers have developed various techniques based on linear matrix inequalities (LMIs) for solving optimization problems over polynomials. The interest for these techniques is mainly motivated by the fact that one can obtain bounds to the global optimum by solving convex optimization problems, and by the fact that the conservatism of these bounds can be decreased in general by suitably increasing the size of the problems.

The objective of this survey is twofold. First, it aims to provide the reader with a significant overview of the LMI techniques that are used in control systems for tackling optimization problems over polynomials. Specifically, the problem of establishing positivity of a polynomial is initially considered, hence describing decomposition in sum of squares and its conservatism aspects related to Hilbert's 17th problem. Then, the problem of establishing positivity of a polynomial under polynomial constraints is addressed, hence illustrating Positivstellensatz, theory of moments, Pólya's theorem, matrix dilation approach, and other techniques for investigating positivity over special sets such as ellipsoids and simplex. Second, this survey aims to provide a collection of the essential problems in control systems where these LMI techniques turn out to be helpful. In particular, the problems of establishing stability and invariance in nonlinear systems, robust stability and robust performance in uncertain systems, stability in time-delay systems, convergence properties in genetic regulatory networks, and optimal strategies in game theory, are reviewed.

The paper is organized as follows. Section II introduces the notation and some preliminaries about polynomials. Sections III-V describe the LMI techniques for positivity, constrained positivity, and positivity over special sets, respectively. Section VI illustrates the application of these LMI techniques in various areas of control systems. Last, Section VII concludes the paper with some final remarks.

\section{PRELIMINARIES}

Let us introduce the notation adopted throughout the paper: - w.r.t., s.t.: with respect to, subject to;

$-X^{\prime}$ : transpose of matrix $X$;

$-X>0$ (respectively, $X \geq 0$ ): symmetric positive definite (respectively, semidefinite) matrix $X$;

$-X \otimes Y:$ Kronecker product of matrices $X$ and $Y$;

$-\operatorname{tr}(X)$ : trace of matrix $X$;

$-\operatorname{co}\{x, y, \ldots\}:$ convex hull of vectors $x, y, \ldots$; 
- $x_{i}$ (respectively, $X_{i, j}$ ): $i$-th (respectively, $(i, j)$-th) entry of vector $x$ (respectively, matrix $X$ ) unless specified otherwise;

$-x^{y}:=x_{1}^{y_{1}} \cdots x_{n}^{y_{n}}$, with $n \times 1$ vectors $x, y$;

- $\|x\|:$ euclidean norm of vector $x$;

$-\nabla f:$ column vector with first-order partial derivatives of $f: \mathbb{R}^{n} \rightarrow \mathbb{R}$

$-\mathbb{N}, \mathbb{R}$, : natural numbers (including zero), real numbers;

$-\mathbb{S}^{n}:=\left\{X \in \mathbb{R}^{n \times n}: X=X^{\prime}\right\}$;

$-0_{n}$ (respectively, $0_{m \times n}$ ): origin of $\mathbb{R}^{n}$ (respectively, $\left.\mathbb{R}^{m \times n}\right)$;

$-I_{n}: n \times n$ identity matrix;

$-\mathbb{R}_{0}^{n}:=\mathbb{R}^{n} \backslash\left\{0_{n}\right\}$.

A function $m: \mathbb{R}^{n} \rightarrow \mathbb{R}$ is a monomial if $m(x)=c_{a} x^{a}$ where $x \in \mathbb{R}^{n}, c_{a} \in \mathbb{R}$ and $a \in \mathbb{N}^{n}$. The quantity $a_{1}+\cdots+a_{n}$ is the degree of $m$. A function $p: \mathbb{R}^{n} \rightarrow \mathbb{R}$ is a polynomial if it is a sum of monomials $m_{1}, m_{2}, \ldots: \mathbb{R}^{n} \rightarrow \mathbb{R}$ with finite degree. The largest degree of the monomials $m_{1}, m_{2}, \ldots$ is the degree of $p$. The set of polynomials $p: \mathbb{R}^{n} \rightarrow \mathbb{R}$ is denoted by $\mathcal{P}$.

A function $P: \mathbb{R}^{n} \rightarrow \mathbb{R}^{r \times r}$ is a (square) matrix polynomial if $P_{i, j} \in \mathcal{P}$ for all $i, j=1, \ldots, r$. The largest degree of the entries of $P$ is the degree of $P$. The set of matrix polynomials $P: \mathbb{R}^{n} \rightarrow \mathbb{R}^{r \times r}$ is denoted by $\mathcal{P}_{r}$.

The quantity $x^{\{d\}} \in \mathbb{R}^{\sigma(n, d)}$ with $x \in \mathbb{R}^{n}$ is a power vector for the polynomials in $\mathcal{P}$ of degree $d$ if it is a vector containing a basis for such polynomials, where $\sigma(n, d)$ is the number of distinct monomials in $x$ of degree not greater than $d$ given by

$$
\sigma(n, d)=\frac{(n+d) !}{n ! d !} .
$$

A simple choice for $x^{\{d\}}$ is a vector whose entries are the monomials in $x$ of degree not greater than $d$. By using power vectors, $p \in \mathcal{P}$ of degree $d$ can be expressed as

$$
p(x)=c^{\prime} x^{\{d\}}
$$

where $c \in \mathbb{R}^{\sigma(n, d)}$. Similarly, $P \in \mathcal{P}_{r}$ of degree $d$ can be expressed as

$$
P(x)=C^{\prime}\left(x^{\{d\}} \otimes I_{r}\right)
$$

where $C \in \mathbb{R}^{r \sigma(n, d) \times r}$.

A particular class of polynomials is represented by the homogeneous polynomials, i.e., polynomials obtained as sum of monomials of the same degree, which are also called forms. Homogeneous polynomials can be expressed as in (2) by using power vectors containing only monomials of the same degree. It is interesting to observe that any polynomial can be viewed as a homogeneous polynomial with one more variable set to 1 . More specifically, by expressing $p(x)=\sum_{i=0}^{d} p_{i}(x)$ where $p_{i} \in \mathcal{P}$ is homogeneous of degree $i$, one has that

$$
p(x)=\left.\hat{p}(y)\right|_{y_{n+1}=1}
$$

where $y=\left(x^{\prime}, 1\right)^{\prime}$ and $\hat{p}(y)$ is the homogeneous polynomial given by

$$
\hat{p}(y)=\sum_{i=0}^{d} p_{i}(x) y_{n+1}^{d-i}
$$

Similarly, homogeneous matrix polynomials are matrices whose entries are homogeneous polynomials.

\section{PositIVITY}

A primary issue in control systems concerns positivity of polynomials. This section introduces a fundamental technique that allows one to investigate this issue via LMI techniques. For conciseness, throughout the paper the adjective "positive" will indicate nonnegativity in the scalar case and positive semidefiniteness in the matrix case (other kinds of positivity will be explicitly defined wherever required).

\section{A. SOS Polynomials}

$p \in \mathcal{P}$ is positive if $p(x) \geq 0$ for all $x \in \mathbb{R}^{n}$. An interesting way of establishing whether $p$ is positive consists of establishing whether $p$ can be written as a sum of squares of polynomials (SOS), i.e.

$$
p(x)=\sum_{i=1}^{k} p_{i}(x)^{2}
$$

for some $p_{1}, \ldots, p_{k} \in \mathcal{P}$. Clearly, any SOS polynomial is positive.

SOS polynomials have been exploited since long time, see for instance the pioneering works [2], [3]

A reason that has motivated the interest for SOS polynomials is that, establishing whether a polynomial is SOS, amounts to solving a convex optimization problem ${ }^{1}$. Indeed, $p \in \mathcal{P}$ of degree $2 d$ can be represented according to the Gram matrix method [4] (also known as square matricial representation (SMR) [5]) via

$$
p(x)=x^{\{d\}^{\prime}}(G+L(\alpha)) x^{\{d\}}
$$

where $G \in \mathbb{S}^{\sigma(n, d)}, L(\alpha)$ is a linear parametrization of the set

$$
\mathcal{L}=\left\{L \in \mathbb{S}^{\sigma(n, d)}: x^{\{d\}^{\prime}} L x^{\{d\}}=0 \forall x \in \mathbb{R}^{n}\right\}
$$

$\alpha \in \mathbb{R}^{\omega(n, 2 d)}$ is a free vector, and $\omega(n, 2 d)$ is the dimension of $\mathcal{L}$ given by

$$
\omega(n, 2 d)=\frac{1}{2} \sigma(n, d)(1+\sigma(n, d))-\sigma(n, 2 d) .
$$

By exploiting this representation, one can establish whether $p$ is SOS as explained in the following result [5], [6].

Theorem 1: $p$ is SOS if and only if there exists $\alpha$ such that

$$
G+L(\alpha) \geq 0 .
$$

The condition (8) is an LMI, and establishing whether an LMI holds for some value of the variables ( $\alpha$ in this case) is an $L M I$ feasibility test, which amounts to solving a convex optimization problem [7]. The matrix $G$ is called Gram matrix (or SMR matrix) of $p$. It is worth observing that $G$ is not unique, and that $G+L(\alpha)$ is a linear matrix function that parametrizes the whole set of Gram matrices of $p$.

\footnotetext{
${ }^{1}$ It is worth observing that the set of positive polynomials is convex analogously to the set of SOS polynomials, however for the former set there is no a tractable characterization contrary to the latter one.
} 
Depending on the monomials present in $p$, the size of the power vector $x^{\{d\}}$ in (5) can be reduced while preserving the validity of Theorem 1 . In fact, the monomials required to write $p$ as SOS can be chosen without loss of generality in the Newton polytope, see [8] for details. This may allow to reduce the complexity of the LMI feasibility test (8).

In the case of matrix polynomials, we say that $P \in \mathcal{P} r$ is positive if $P(x) \geq 0$ for all $x \in \mathbb{R}^{n}$. Positivity of matrix polynomials can be investigated by extending the concept of SOS. Specifically, $P$ is a SOS matrix polynomial if there exist $P_{1}, \ldots, P_{k} \in \mathcal{P}_{r}$ such that

$$
P(x)=\sum_{i=1}^{k} P_{i}(x)^{\prime} P_{i}(x) .
$$

Equivalently, $P$ is SOS if and only if the polynomial $y^{T} P(x) y$ is SOS in the extended variable $\left(x^{\prime}, y^{\prime}\right)^{\prime}$, see e.g., [9]. Clearly, any SOS matrix polynomial is positive.

A symmetric matrix polynomial $P \in \mathcal{P}_{r}$ of degree $2 d$ can be represented through a suitable extension of (5) as

$$
P(x)=\left(x^{\{d\}} \otimes I_{r}\right)^{\prime}(G+L(\alpha))\left(x^{\{d\}} \otimes I_{r}\right)
$$

where $G \in \mathbb{S}^{r \sigma(n, d)}, L(\alpha)$ is a linear parametrization of the set

$$
\begin{aligned}
\mathcal{L}= & \left\{L \in \mathbb{S}^{r \sigma(n, d)}:\left(x^{\{d\}} \otimes I_{r}\right)^{\prime} L\left(x^{\{d\}} \otimes I_{r}\right)=0_{r \times r}\right. \\
& \left.\forall x \in \mathbb{R}^{n}\right\}
\end{aligned}
$$

$\alpha \in \mathbb{R}^{\omega(n, 2 d, r)}$ is a free vector, and $\omega(n, 2 d, r)$ is the dimension of $\mathcal{L}$ given by

$$
\begin{aligned}
\omega(n, 2 d, r)= & \frac{1}{2} r(\sigma(n, m)(r \sigma(n, m)+1) \\
& -(r+1) \sigma(n, 2 m)) .
\end{aligned}
$$

Similarly to the scalar case, $P$ is SOS if and only if there exists $\alpha$ such that the LMI $G+L(\alpha) \geq 0$ holds [10], [11]. In the sequel, the set of SOS polynomials in $\mathcal{P}$ (respectively, SOS matrix polynomials in $\mathcal{P}_{r}$ ) will be denoted by $\mathcal{P}^{S O S}$ (respectively, $\left.\mathcal{P}_{r}^{S O S}\right)$.

SOS polynomials can be used also to establish strict positivity of polynomials. A simple strategy is the following: $P \in \mathcal{P}_{r}$ satisfies $P(x)>0$ for all $x \in \mathbb{R}^{n}$ if there exists $\varepsilon>0$ such that

$$
P(x)-\varepsilon I_{r} \in \mathcal{P}_{r}^{S O S}
$$

For homogeneous polynomials, strict positivity does not involve the origin since the polynomial vanishes at this point. A strategy is hence the following: a homogeneous $P \in \mathcal{P}_{r}$ satisfies $P(x)>$ 0 for all $x \in \mathbb{R}_{0}^{n}$ if there exists $\varepsilon>0$ such that

$$
P(x)-\varepsilon\|x\|^{2 d} I_{r} \in \mathcal{P}_{r}^{S O S} .
$$

Alternatively, one can simply define a power vector with only monomials of degree $d$ in $x$, hence requiring that $G+L(\alpha)>0$. Before proceeding it is worth mentioning that the linear matrix function $L(\alpha)$, which allows to equivalently express via an LMI feasibility test the SOS condition for either a polynomial or a matrix polynomial, can be systematically constructed. Indeed, $L(\alpha)$ is a linear parametrization of a linear subspace with finite dimension, and hence can be built by using standard algorithms of linear algebra. The reader is referred to [12] for algorithms about the construction of $G$ and $L(\alpha)$.

\section{B. Hilbert's 17th Problem}

The interest for SOS polynomials has been motivated not only by the convexity of the optimization problem that one needs to solve for establishing whether a polynomial is SOS, but also by some available results that characterize the gap between positive polynomials and SOS polynomials.

The story goes back to Hilbert's 17th problem (see e.g., [13] and references therein), which asks whether all positive polynomials are sums of squares of rational functions. Specifically, in 1888 Hilbert found an answer to a closely related question: not all positive polynomials are SOS.

Let us refer to polynomials that are positive but not SOS as PNS polynomials. One of the first examples of PNS polynomials was given by Motzkin in 1967. Motzkin's polynomial is a polynomial in 2 variables of degree 6 given by

$$
p_{M o t}(x)=x_{1}^{4} x_{2}^{2}+x_{1}^{2} x_{2}^{4}-3 x_{1}^{2} x_{2}^{2}+1 \text {. }
$$

See [13], [14] and references therein for other examples of PNS polynomials. As explained in the following result, PNS polynomials do not exist depending on the number of variables and on the degree [15].

Theorem 2: Consider $p \in \mathcal{P}$ of degree $2 d$, and suppose that $(n, 2 d) \in \mathcal{E}$ where

$$
\mathcal{E}=\{(n, 2), n \in \mathbb{N}\} \cup\{(1,2 d), d \in \mathbb{N}\} \cup\{(2,4)\} .
$$

Then, $p$ is positive if and only if it is SOS.

Further results are available on the gap between positive polynomials and SOS polynomials. In particular, a result analogous to Theorem 2 establishes that a homogeneous $p \in \mathcal{P}$ of degree $2 d$ with $(n, 2 d) \in \mathcal{E}$ satisfies $p(x)>0$ for all $x \in \mathbb{R}_{0}^{n}$ if and only if it admits a positive definite Gram matrix (see [12] and references therein). Moreover, SOS polynomials are dense among positive polynomials, but they become sparse by fixing the degree and increasing the number of variables [16], [17]. Also, in [14] it is proved that any PNS polynomial is the vertex of a cone of PNS polynomials, and a parametrization of the set of PNS polynomials is proposed. The following example is built according to this parametrization:

$$
\begin{aligned}
p(x)=4\left(x_{1}^{6}\right. & \left.+x_{2}^{6}+1\right)-19\left(x_{1}^{4} x_{2}^{2}+x_{2}^{4}+x_{1}^{2}\right)-30 x_{1}^{2} x_{2}^{2} \\
& +29\left(x_{1}^{4}+x_{2}^{4} x_{1}^{2}+x_{2}^{2}\right)-\delta\left(x_{1}^{2}+x_{2}^{2}+1\right)^{3}
\end{aligned}
$$

which is a PNS polynomial for all $\delta \in(0,0.436)$ [12].

In 1927, Artin proved the following fundamental result, which provides a positive answer to Hilbert's 17th problem.

Theorem 3: Any $p \in \mathcal{P}$ is positive if and only if there exist $s_{1}, s_{2} \in \mathcal{P}^{S O S}$ such that

$$
p(x)=\frac{s_{1}(x)}{s_{2}(x)} .
$$


Observe that, for a fixed degree of $s_{2}$, the condition (18) can be expressed via an LMI feasibility test by requiring that $s_{2}$ and $p_{1} s_{2}$ are SOS with $s_{2} \neq 0$. Specifically, let $G_{2}$ and $G_{3}\left(G_{2}\right)+$ $L(\alpha)$ be a Gram matrix of $s_{2}$ and a linear parametrization of the Gram matrices of $p_{1} s_{2}$ respectively, and observe that $G_{3}\left(G_{2}\right)$ depends linearly on $G_{2}$. Then, $s_{2}$ and $p_{1} s_{2}$ are SOS (with $s_{2} \neq$ 0 ) if and only if there exist $G_{2}$ and $\alpha$ such that

$$
G_{2} \geq 0 \text { and } G_{3}\left(G_{2}\right)+L(\alpha) \geq 0 \text { and } \operatorname{tr}\left(G_{2}\right)=1
$$

which is an LMI feasibility test (the constraint $\operatorname{tr}\left(G_{2}\right)=1$ is introduced without loss of generality in order to guarantee that $s_{2} \neq 0$ ).

Hilbert's 17th problem has been formulated also in the case of matrix polynomials. In particular, a generalization of Artin's result has been given in [18], which says that $P \in \mathcal{P}_{r}$ is positive if and only if it can be expressed as a sum of squares of symmetric matrices with entries in $\mathbb{R}^{n}$.

The following theorem provides a result analogous to Theorem 2 in the case of matrix polynomials [4].

Theorem 4: Suppose $n=1$. Then, $P \in \mathcal{P}_{r}$ is positive if and only if it is SOS.

\section{Constrained Positivity}

The previous section has considered the problem of establishing whether a polynomial is positive. This section introduces some key techniques that allow one to investigate positivity in the presence of constraints.

\section{A. Scalar Case}

Constrained positivity of polynomials can be investigated via techniques "after" of polynomials known as Positivstellensatz. In order to describe these techniques, let us introduce the following basic definitions (see, e.g., [6], [19], [20] for details). The multiplicative monoid generated by polynomials $p_{1}, \ldots, p_{k} \in \mathcal{P}$, denoted by $\mathcal{M}\left(p_{1}, \ldots, p_{k}\right)$, is the set of finite products of these polynomials. The cone generated by polynomials $p_{1}, \ldots, p_{k} \in \mathcal{P}$ is the set

$$
\begin{aligned}
\mathcal{C}\left(p_{1}, \ldots, p_{k}\right)= & \left\{s_{0}+\sum_{i=1}^{j} s_{i} q_{i}: s_{0}, \ldots, s_{j} \in \mathcal{P}^{S O S},\right. \\
& \left.q_{1}, \ldots, q_{j} \in \mathcal{M}\left(p_{1}, \ldots, p_{k}\right)\right\} .
\end{aligned}
$$

Last, the ideal generated by polynomials $p_{1}, \ldots, p_{k} \in \mathcal{P}$ is the set

$$
\mathcal{I}\left(p_{1}, \ldots, p_{k}\right)=\left\{\sum_{i=1}^{k} r_{i} p_{i}: r_{1}, \ldots, r_{k} \in \mathcal{P}\right\}
$$

The following Positivstellensatz is proposed in [21].
Theorem 5: Let $f_{1}, \ldots, f_{k}, g_{1}, \ldots, g_{l}$, and $h_{1}, \ldots, h_{m}$ belong to $\mathcal{P}$. Define the set

$$
\begin{gathered}
\mathcal{X}=\left\{x \in \mathbb{R}^{n}: f_{1}(x) \geq 0, \ldots, f_{k}(x) \geq 0\right. \\
\text { and } g_{1}(x)=\cdots=g_{l}(x)=0 \\
\text { and } \left.h_{1}(x) \neq 0, \ldots, h_{m}(x) \neq 0\right\} .
\end{gathered}
$$

Then

$$
\begin{aligned}
\mathcal{X}=\emptyset \Longleftrightarrow \exists & f \in \mathcal{C}\left(f_{1}, \ldots, f_{k}\right), g \in \mathcal{I}\left(g_{1}, \ldots, g_{l}\right), \\
& h \in \mathcal{M}\left(h_{1}, \ldots, h_{m}\right): \\
& f(x)+g(x)+h(x)^{2}=0 .
\end{aligned}
$$

A stronger version of the Positivstellensatz is as follows [22]. Theorem 6: Let $f_{1}, \ldots, f_{k} \in \mathcal{P}$ be such that the set

$$
\mathcal{K}=\left\{x \in \mathbb{R}^{n}: f_{1}(x) \geq 0, \ldots, f_{k}(x) \geq 0\right\}
$$

is compact. Let $p \in \mathcal{P}$. Then

$$
p(x)>0 \forall x \in \mathcal{K} \Longleftrightarrow p \in \mathcal{C}\left(f_{1}, \ldots, f_{k}\right) .
$$

A further stronger condition, proposed in [23], is stated in the following result.

Theorem 7: Let $f_{1}, \ldots, f_{k} \in \mathcal{P}$ be polynomials of even degree such that their highest degree forms do not have common zeros in $\mathbb{R}_{0}^{n}$, and such that the set $\mathcal{K}$ in (24) is compact. Let $p \in \mathcal{P}$. Then

$$
\begin{aligned}
& p(x)>0 \forall x \in \mathcal{K} \Longleftrightarrow \exists s_{0}, \ldots, s_{k} \in \mathcal{P}^{S O S}: \\
& p=s_{0}+\sum_{i=1}^{k} s_{i} f_{i} .
\end{aligned}
$$

The conditions described in Theorems 5-7 can be expressed via $\mathrm{LMI}$ feasibility tests for fixed degree of the multipliers. As an example, let $a_{1}, \ldots, a_{k}, b_{1}, \ldots, b_{l}$ and $p$ belong to $\mathcal{P}$, and consider the problem of establishing whether the following condition holds:

$$
\begin{aligned}
p(x) \geq 0 \forall x \in \mathbb{R}^{n}: & a_{i}(x)=0 \forall i=1, \ldots, k \\
& \text { and } b_{j}(x) \geq 0 \forall j=1, \ldots, l .
\end{aligned}
$$

It is easy to see that this condition holds if the following condition holds, which is obtained from Theorem 5:

$$
\begin{aligned}
& \exists r_{1}, \ldots, r_{k} \in \mathcal{P}, s_{0}, \ldots, s_{l} \in \mathcal{P}^{S O S}: \\
& p=\sum_{i=1}^{k} r_{i} a_{i}+\sum_{i=1}^{l} s_{i} b_{i}+s_{0} .
\end{aligned}
$$

For fixed degree of $r_{1}, \ldots, r_{k}, s_{0}, \ldots, s_{l}$, the condition above can be rewritten as an LMI feasibility test by exploiting the technique in Section III-A, see also the works [6], [24]. 
It is worth mentioning that the Positivstellensatz can be regarded as a generalization of the S-procedure, where the polynomials defining the problem are quadratic and the multipliers are constants [7].

\section{B. Matrix Case}

Positivstellensatz can be formulated not only for polynomials but also for matrix polynomials. Let us define the function $\operatorname{tr}_{r}$ : $\mathbb{R}^{q r \times q r} \rightarrow \mathbb{R}^{r \times r}$ as

$$
\operatorname{tr}_{r}(C)=\left(\begin{array}{ccc}
\operatorname{tr}\left(C_{11}\right) & \cdots & \operatorname{tr}\left(C_{1 r}\right) \\
\vdots & \ddots & \vdots \\
\operatorname{tr}\left(C_{r 1}\right) & \cdots & \operatorname{tr}\left(C_{r r}\right)
\end{array}\right)
$$

where $C_{i j} \in \mathbb{R}^{q \times q}$ is the $(i, j)$-th block of the matrix $C \in$ $\mathbb{R}^{q r \times q r}$. The following result investigates positivity of matrix polynomials on compact semi-algebraic sets [25].

Theorem 8: Let $F \in \mathcal{P}_{q}$ and define

$$
\mathcal{F}=\left\{x \in \mathbb{R}^{n}: F(x) \leq 0\right\} .
$$

Suppose that there exists $c \in \mathbb{R}$ and $S_{1} \in \mathcal{P}_{q}^{S O S}$ such that

$$
c^{2}-\|x\|^{2}+\operatorname{tr}\left(S_{1}(x) F(x)\right) \in \mathcal{P}^{S O S} .
$$

Let $P \in \mathcal{P}_{r}$. Then

$$
P(x) \geq 0 \forall x \in \mathcal{F}
$$

if and only if there exists $S_{2} \in \mathcal{P}_{q r}^{S O S}$ such that

$$
P(x)+\operatorname{tr}_{r}\left(S_{2}(x)\left(I_{r} \otimes F(x)\right)\right) \in \mathcal{P}_{r}^{S O S} .
$$

The conditions (31) and (33) can be expressed via LMI feasibility tests for fixed degrees of $S_{1}$ and $S_{2}$ by exploiting the technique in Section III-A. The result provided in Theorem 8 is obtained in [26], [27] for the case $r=1$ (i.e., $P$ is a polynomial rather than a matrix polynomial). Also, this result is obtained in [23] for the case of diagonal $F$.

Another technique for positivity of polynomials on compact semi-algebraic sets described by matrix polynomial inequalities is based on the theory of moments, which is dual to the SOS technique. For clarity of description, we present here the use of this technique for determining the minimum of a polynomial under matrix polynomial inequalities, as it is given in [28]. Let us introduce the following definitions. Let $y \in \mathbb{R}^{l}$ be a variable with entries denoted by $y_{a}$, where $a \in \mathbb{N}^{n}$ is a multi-index and $l \in \mathbb{N}$ will be specified in the sequel. An example for the case $n=2$ is

$$
y=\left(y_{00}, y_{10}, y_{01}, y_{20}, y_{11}, y_{02}\right)^{\prime} .
$$

For $P \in \mathcal{P}_{r}$ expressed as

$$
P(x)=\sum_{a \in \mathbb{N}^{n}} C_{a} x^{a}
$$

with matrices $C_{a} \in \mathbb{R}^{r \times r}$ indexed by $a$, define the function

$$
L(y, P)=\sum_{a \in \mathbb{N}^{n}} C_{a} y_{a} .
$$

For $F \in \mathcal{P}_{r}$ of degree $2 d$ or $2 d-1$, and for an integer $k \geq d$, define the matrix

$$
\begin{aligned}
M_{k-d}(y, F) & =L(y, \hat{F}), \\
\hat{F}(x) & =\left(x^{\{k-d\}} x^{\{k-d\}^{\prime}}\right) \otimes F(x)
\end{aligned}
$$

where the power vector $x^{\{k-d\}}$ is chosen so that all its entries are monomials.

Theorem 9: Consider the optimization problem

$$
m=\min _{x \in \mathbb{R}^{n}} f(x) \text { s.t. } F(x) \geq 0
$$

where $f \in \mathcal{P}$ has degree $2 c$ or $2 c-1$, and $F \in \mathcal{P}_{r}$ has degree $2 d$ or $2 d-1$. For any integer $k \geq \max \{c, d\}$ define

$$
\begin{array}{ll}
m^{(k)}=\min _{y \in \mathbb{R}^{l}} L(y, f) \quad \text { s.t. } & y_{0_{n}}=1, M_{k}(y, 1) \geq 0, \\
& M_{k-d}(y, F) \geq 0 .
\end{array}
$$

Then

$$
m^{(k)} \leq m
$$

Moreover, if there exist $s \in \mathcal{P}^{S O S}$ and $S \in \mathcal{P}_{r}^{S O S}$ such that $\left\{x \in \mathbb{R}^{n}: s(x)+\operatorname{tr}(S(x) F(x)) \geq 0\right\}$ is compact, then

$$
\lim _{k \rightarrow \infty} m^{(k)}=m \text {. }
$$

Theorem 9 provides a sequence of lower bounds $m^{(k)}$ to the solution $m$ of (38). Since $L(y, f), M_{k}(y, 1)$ and $M_{k-d}(y, F)$ are linear in $y$, computing $m^{(k)}$ amounts to minimizing a linear function subject to LMI constraints, which is a convex optimization problem known as semidefinite program $(S D P)$. The matrices $M_{k}(y, 1)$ and $M_{k-d}(y, F)$ are known as truncated moment matrix and localizing moment matrix, respectively. The length $l$ of the vector $y$ is given by the number of monomials in $x$ of degree not greater than $2 k$, and it is hence $l=\sigma(n, 2 k)$.

As discussed in [28], optimality can be detected a posteriori through a simple rank condition, and the global minimizers of (38) can be computed via linear algebra operations whenever this rank condition holds. Also, [28] describes an interesting application of Theorem 9 to static output feedback control synthesis. Last, Theorem 9 is obtained in [29] for the case of diagonal $F$.

\section{Positivity Over Special Sets}

Often control problems require to investigate positivity of polynomials on special sets, for which dedicated results have been derived as described in the sequel. 


\section{A. Positivity Over the Simplex}

Let us start by considering the case where the variable of a polynomial is constrained in the simplex, i.e., the set defined by

$$
\mathcal{U}_{n}=\left\{x \in \mathbb{R}^{n}: \sum_{i=1}^{n} x_{i}=1, x_{i} \geq 0\right\} .
$$

The following result characterizes positivity of homogeneous matrix polynomials over the simplex [30].

Theorem 10: Any homogeneous $P \in \mathcal{P}_{r}$ satisfies

$$
P(x)>0 \quad \forall x \in \mathcal{U}_{n}
$$

if and only if there exists $k \in \mathbb{N}$ such that all the matrix coefficients of $P(x)\left(\sum_{i=1}^{n} x_{i}\right)^{k}$ are positive definite.

For any fixed $k$, Theorem 10 provides a sufficient condition for (43) by simply testing the positive definiteness of some matrices. Moreover, this condition is also necessary by using a sufficiently large $k$. For $r=1$, Theorem 10 coincides with Pólya's theorem, which characterizes positivity of homogeneous polynomials over the simplex. It is worth mentioning that several bounds on the value of $k$ required for achieving necessity in Pólya's theorem have been found, which however require the knowledge of the minimum of $p$ over the simplex, see for instance [31].

Another result for positivity of matrix polynomials over the simplex is given in [10]. Specifically, let $P \in \mathcal{P}_{r}$, and consider the problem of establishing whether (43) holds. Let $d$ be the degree of $P$, and rewrite $P$ as

$$
P(x)=\sum_{i=0}^{d} H_{i}(x)
$$

for homogeneous $H_{i} \in \mathcal{P}_{r}$ of degree $i$. Let us define the homogeneous matrix polynomial

$$
H(x)=\sum_{i=0}^{d} H_{i}(x)\left(\sum_{i=1}^{n} x_{i}\right)^{d-i} .
$$

Theorem 11: Any $P \in \mathcal{P}_{r}$ satisfies (43) if and only if

$$
\hat{H}(x)>0 \forall x \in \mathbb{R}_{0}^{n}, \quad \hat{H}(x)=\left.H(y)\right|_{y=\left(x_{1}^{2}, \ldots, x_{n}^{2}\right)^{\prime}} .
$$

Theorem 11 provides a condition equivalent to (43) where the variable $x$ is not constrained in the simplex. As a consequence, a sufficient condition for (43) can be given immediately via an LMI feasibility test by as explained at the end of Section III-A "after" feasibility test.

\section{B. Positivity Over Ellipsoids}

Here we consider the problem of establishing whether a polynomial is positive over an ellipsoid, i.e.,

$$
p(x)>0 \forall x \in \mathbb{R}^{n}: x^{\prime} Q x=c
$$

where $p \in \mathcal{P}, Q \in \mathbb{S}^{n}$ and $c \in \mathbb{R}$, with $Q>0$ and $c \geq 0$. An interesting strategy reformulates the above problem into the problem of establishing whether a homogeneous polynomial is positive [5]. Specifically, let $d$ be the degree of $p$, and rewrite $p(x) p(-x)$ as

$$
p(x) p(-x)=\sum_{i=0}^{d} \bar{p}_{2 i}(x)
$$

where $\bar{p}_{2 i}(x) \in \mathcal{P}$ is a homogeneous polynomial of degree $2 i$. Let us define the homogeneous polynomial of degree $2 d$

$$
h(x)=\sum_{i=0}^{d} \bar{p}_{2 i}(x)\left(\frac{x^{\prime} Q x}{c}\right)^{d-i} .
$$

Theorem 12: Let us assume that

$$
p\left(x_{\text {any }}(c)\right)>0 x_{\text {any }}(c)=\sqrt{\frac{c}{Q_{1,1}}}(1,0, \ldots, 0)^{\prime} .
$$

Then, (47) holds if and only if $h(x)>0$ for all $x \in \mathbb{R}_{0}^{n}$.

Theorem 12 is interesting because it provides a necessary and sufficient condition for (47) without introducing unknown multipliers. Let us observe that the assumption (50) is not restrictive because, if it does not hold, then one immediately concludes that (47) cannot hold. A sufficient condition for the positivity condition on $h$ obtained in Theorem 12 can be obtained via an LMI feasibility test as explained at the end of Section III-A "after" feasibility test. This LMI condition is also necessary if $(n, 2 d) \in \mathcal{E}$ [12], [32].

\section{Positivity Over Convex Polytopes}

Another interesting case concerns positivity of matrix polynomials over convex polytopes, for which a technique known as matrix dilation approach and slack variable approach can be used. This technique has been introduced by various authors, see, e.g., [33], [34] and references therein. Specifically, let us express a symmetric $P \in \mathcal{P}_{r}$ as

$$
P(x)=M(x)^{\prime} G M(x)
$$

where $G$ is a symmetric matrix, and $M$ is a matrix polynomial. Moreover, let $H$ be a matrix polynomial such that

$$
\left\{\begin{array}{l}
M(x)^{\prime} H(x)=0 \\
(M(x) H(x)) \text { is square and nonsingular } \\
H(x) \text { is affine linear. }
\end{array}\right.
$$

Theorem 13: Let $P \in \mathcal{P}_{r}$ and $v^{(1)}, \ldots, v^{(k)} \in \mathbb{R}^{n}$ be given. Let $G, H$ be defined as in (51)-(52). Then

$$
P(x) \geq 0 \forall x \in \operatorname{co}\left\{v^{(1)}, \ldots, v^{(k)}\right\}
$$

if there exists a matrix $W$ such that

$$
G+H(x) W^{\prime}+W H(x)^{\prime} \geq 0 \quad \forall x \in\left\{v^{(1)}, \ldots, v^{(k)}\right\} .
$$

See also [35] for a comparison of this technique with the SOS approach presented in Section III-A.

\section{APPLICATIONS}

This section briefly reviews some relevant applications in control systems of the techniques introduced in Sections III-V. It is worth mentioning that some of these techniques are 
implemented in the MATLAB toolboxes GloptiPoly [36], SOSTOOLS [37] and YALMIP [38].

\section{A. Nonlinear System Stability}

A fundamental application of LMI techniques for optimization problems over polynomials is in nonlinear systems, and consists of establishing whether an equilibrium point is globally asymptotically stable. Let us start by considering

$$
\left\{\begin{array}{l}
\dot{x}(t)=f(x(t)) \\
f\left(0_{n}\right)=0_{n}
\end{array}\right.
$$

where $t \in \mathbb{R}$ is the time, $x \in \mathbb{R}^{n}$ is the state, $f: \mathbb{R}^{n} \rightarrow \mathbb{R}^{n}$ is nonlinear, and the origin is the equilibrium point of interest. Global asymptotic stability of the origin can be investigated by looking for a Lyapunov function $v: \mathbb{R}^{n} \rightarrow \mathbb{R}$ such that (see, e.g., [39] and references therein)

$$
\left\{\begin{array}{l}
v\left(0_{n}\right)=0, v(x)>0 \forall x \in \mathbb{R}_{0}^{n}, v(x) \rightarrow \infty \text { as }\|x\| \rightarrow \infty \\
-\nabla v(x)^{\prime} f(x)>0 \forall x \in \mathbb{R}_{0}^{n} .
\end{array}\right.
$$

This condition can be investigated through the technique in Section III-A in the case that $f$ and $v$ are polynomial [6]. Specifically, let us assume that the system (55) linearized in the origin is asymptotically stable. Then, one can ensure the fulfillment of the condition (56) by requiring that the polynomials

$$
v(x)-\varepsilon\|x\|^{2},-\left(\nabla v(x)^{\prime} f(x)+\varepsilon\|x\|^{2}\right)
$$

are SOS for some $\varepsilon>0$, which amounts to solving an LMI feasibility test where the variables are $\varepsilon$, the coefficients of $v$, and vectors such as $\alpha$ in (8). A similar strategy can be adopted in the case that the system (55) linearized in the origin is only marginally stable, see [40] for details.

\section{B. Set Invariance for Nonlinear Systems}

Another fundamental problem in nonlinear systems consists of determining invariant sets, i.e., sets from which no trajectory can escape. Indeed, let us consider again (55). A set $\mathcal{I} \subset \mathbb{R}^{n}$ is positively invariant for (55) if

$$
x(t) \in \mathcal{I} \forall t \geq 0 \forall x(0) \in \mathcal{I} .
$$

In addition, if $x$ converges to the equilibrium point of interest as $t \rightarrow \infty$ for all $x(0) \in \mathcal{I}$, then $\mathcal{I}$ is a strictly positively invariant set.

Positively invariant sets can be characterized by using Lyapunov functions, see e.g., [39] and references therein. For instance, if there exists $v: \mathbb{R}^{n} \rightarrow \mathbb{R}$ such that

$$
\left\{\begin{array}{l}
v\left(0_{n}\right)=0, v(x)>0 \forall x \in \mathbb{R}_{0}^{n}, v(x) \rightarrow \infty \text { as }\|x\| \rightarrow \infty \\
-\nabla v(x)^{\prime} f(x)>0 \forall x \in \mathcal{V}(1) \backslash\left\{0_{n}\right\}
\end{array}\right.
$$

where $\mathcal{V}(1)$ is the unitary sublevel set of $v$ defined by

$$
\mathcal{V}(c)=\{x: v(x) \leq c\}
$$

then $\mathcal{V}(1)$ is a strictly positively invariant set for (55).

Condition (58) can be investigated through the techniques in Section IV-A and Section IV-B in the case that $f$ and $v$ are polynomial analogously to the stability investigation in Section VI-A. Let us observe that these conditions amount to solving an LMI feasibility test if $v$ is fixed, whereas they amount to solving a bilinear matrix inequality (BMI) feasibility test if $v$ is variable 2 . Also, if $v$ is quadratic, one can use the technique in Section V-B.

This strategy for determining invariant sets has been proposed starting from [5], [6], [41] which consider the estimation of the domain of attraction of equilibrium points, see also [42]-[44] and references therein for recent contributions. It is useful mentioning that if $f$ is polynomial, then it is not restrictive to assume that also $v$ is polynomial for exponential stability on bounded domains [45].

\section{Robust Stability}

Linear systems affected by structured uncertainty are an important area of automatic control. A model often used in the literature for these systems is given by

$$
\left\{\begin{array}{l}
\dot{x}(t)=A(\theta(t)) x(t) \\
\theta(t) \in \mathcal{U}_{q}
\end{array}\right.
$$

where $\theta: \mathbb{R} \rightarrow \mathbb{R}^{q}$ is the uncertainty, $\mathcal{U}_{q}$ is the simplex in (42), and $A: \mathbb{R}^{q} \rightarrow \mathbb{R}^{n \times n}$ is linear. The robust stability problem consists of establishing whether the equilibrium point $x=0_{n}$ is asymptotically stable for all admissible values of the uncertainty.

In the case of time-invariant uncertainty, i.e., $\theta$ is constant w.r.t. $t$, this problem can be addressed by using homogeneous parameter-dependent quadratic Lyapunov functions. Such a function can be written as

$$
v(x, \theta)=x^{\prime} P(\theta) x
$$

where $P: \mathbb{R}^{q} \rightarrow \mathbb{S}^{n}$ is a homogeneous matrix polynomial. It has been proved in [10] that (60) is asymptotically stable for timeinvariant uncertainty if and only if there exists a homogeneous matrix polynomial $P$ of degree not greater than a known value and satisfying

$$
\left.\begin{array}{c}
P(\theta)>0 \\
-P(\theta) A(\theta)-A(\theta)^{\prime} P(\theta)>0
\end{array}\right\} \forall \theta \in \mathcal{U}_{q} .
$$

One can get rid of the constraint $\theta \in \mathcal{U}_{q}$ as explained in Section V-A, and the problem boils down to check the positive definiteness of homogeneous matrix polynomials. This can be tackled by using the technique in Section III-A, which amounts to solving an LMI feasibility test where the variables are the coefficients of $P$ and the parameters $\alpha$ required to test the SOS condition. Although the use of LMIs provides a sufficient condition for any fixed degree of $P$, it is interesting to observe that nonconservatism is achieved for sufficiently large degree of $P$, see [12] and references therein.

Another technique for investigating (62) via Lyapunov functions with homogeneous dependence on the parameters has been proposed in [46] based on the direct use of Pólya's theorem in Section V-A. In [47] the use of Lyapunov functions with polynomial dependence has been proposed for the case of uncertainty constrained in a hypercube, which can be converted to the simplex model. Other strategies consist of adopting the technique

${ }^{2}$ Solving a BMI feasibility test amounts in general to solving a nonconvex optimization problem. 
presented in Section V-C, see e.g., [48], and $D / G$ scaling, see e.g., [49]. Last, asymptotically stability for time-invariant uncertainty of systems like (60) can be investigated through techniques based on positive polynomials also by adopting non-Lyapunov approaches, for example through Hermite's matrices [50] and Hurwitz's determinants [12].

In the case the uncertainty in (60) is time-varying, i.e., $\theta$ depends on $t$, the robust stability problem can be addressed by using homogeneous polynomial Lyapunov functions that have been proposed in [51], [52]. Denoting the degree of such functions by $2 d$, they can be written as

$$
v(x)=x^{\{d\}^{\prime}} P x^{\{d\}}
$$

where $x^{\{d\}}$ is a power vector containing only monomials of degree $d$, and $P$ is a symmetric matrix of suitable dimension. The origin of (60) is asymptotically stable for time-varying uncertainty if there exist $d$ and $P$ such that

$$
\left.\begin{array}{r}
x^{\{d\}^{\prime}} P x^{\{d\}}>0 \\
-x^{\{d\}^{\prime}}\left(P A_{\times}(\theta)+A_{\times}(\theta)^{\prime} P\right) x^{\{d\}^{\prime}}>0
\end{array}\right\} \quad \forall \theta \in \operatorname{ver}\left(\mathcal{U}_{q}\right)
$$

where $A_{\times}(\theta)$ is the extended matrix of $A(\theta)$ defined by the relation

$$
\frac{d x^{\{m\}}}{d t}=A_{\times}(\theta) x^{\{m\}} .
$$

The condition (64) amounts to checking the positive definiteness of a finite number of homogeneous polynomials, in particular $v$ and its derivative calculated at each vertex of $\mathcal{U}_{q}$, and hence can be tackled with the technique in Section III-A [53]. It is worth mentioning that homogeneous polynomial Lyapunov functions are nonconservative for this robust stability problem [54], and at present it is conjectured that the LMI condition derived from (64) may be nonconservative as well for some sufficiently large $d$. See also [12] and references therein for the application of this technique to uncertain systems in linear fractional representation (LFR) form.

In the case of time-varying uncertainty with bounds on the variation rate, the robust stability problem for system (60) can be addressed via a suitable combination of the methods just described for time-invariant and time-varying uncertainty, in particular by using homogeneous parameter-dependent homogeneous polynomial Lyapunov functions. See e.g., [12] and references therein.

\section{Robust Performance}

Another important issue in uncertain systems consists of establishing a robust performance, i.e., the worst-case value of a performance index such as the $\mathcal{H}_{\infty}$ norm or the $\mathcal{H}_{2}$ norm. In order to describe this problem, let us introduce the system

$$
\left\{\begin{array}{l}
\dot{x}(t)=A(\theta) x(t)+B(\theta) u(t) \\
y(t)=C(\theta) x(t)+D(\theta) u(t) \\
G(\theta) \leq 0
\end{array}\right.
$$

where $x \in \mathbb{R}^{n}$ is the state, $u \in \mathbb{R}^{r}$ is the input, $y \in \mathbb{R}^{p}$ is the output, and the functions $A, B, C, D$ are affine linear of suitable dimension. The vector $\theta \in \mathbb{R}^{q}$ is the uncertainty (for simplicity supposed constant w.r.t. $t$ ), whose set of admissible values is defined by the inequality $G(\theta) \leq 0$, with $G: \mathbb{R}^{q} \rightarrow \mathbb{S}^{w}$ matrix polynomial.

Let us start by considering the estimation of the robust $\mathcal{H}_{\infty}$ performance of (66), which is defined as

$$
\gamma_{\infty}^{*}=\sup _{\theta: G(\theta) \leq 0}\|H(s, \theta)\|_{\infty}
$$

where $H(s, \theta)$ is the transfer matrix from $u$ to $y$ parameterized by $\theta$, and $\|H(s, \theta)\|_{\infty}$ is its $\mathcal{H}_{\infty}$ norm. This problem can be addressed by using polynomially parameter-dependent quadratic Lyapunov functions. Indeed, an upper bound of $\gamma_{\infty}^{*}$ is given by

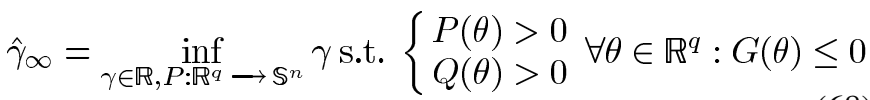

where $P$ is a matrix polynomial, and

$$
\begin{aligned}
Q(\theta)= & -\left(\begin{array}{cc}
P(\theta) A((\theta))+A(\theta)^{\prime} P(\theta) & P(\theta) B(\theta) \\
* & \\
& -I_{r}
\end{array}\right) \\
& -\frac{1}{\gamma^{2}}\left(\begin{array}{cc}
C(\theta)^{\prime} C(\theta) & C(\theta)^{\prime} D(\theta) \\
* & D(\theta)^{\prime} D(\theta)
\end{array}\right) .
\end{aligned}
$$

By requiring that $P$ and $Q$ are SOS, an upper bound to $\hat{\gamma}_{\infty}$ (and hence to $\gamma_{\infty}^{*}$ ) is readily obtained by solving an SDP. This technique has been presented in [25], [30], [55], which also propose tests for establishing exactness of the upper bound. See also [56] which provides a specialization of this technique to discrete-time uncertain systems.

The case of robust $\mathcal{H}_{2}$ performance can be similarly addressed. Indeed, with $D(\theta)=0$, an upper bound to this robust performance is given by

$$
\begin{aligned}
& \hat{\gamma}_{2}=\underset{\gamma \in \mathbb{R}, P: \mathbb{R}^{q} \rightarrow \inf ^{n}, W: \mathbb{R}^{q} \rightarrow \mathbb{S}^{r}}{ } \gamma \\
& \text { s.t. }\left\{\begin{array}{l}
\gamma-\operatorname{tr}(W(\theta))>0 \\
W(\theta)-B(\theta)^{\prime} P(\theta) B(\theta)>0 \\
-P(\theta) A(\theta)-A(\theta)^{\prime} P(\theta)-C(\theta)^{\prime} C(\theta)>0 \\
\forall \theta \in \mathbb{R}^{q}: G(\theta) \leq 0
\end{array}\right.
\end{aligned}
$$

where $P$ and $W$ are matrix polynomials.

Possible extensions include time-varying uncertainties, which can be handled analogously to the case of robust stability analysis in Section VI-D. Also, if the uncertainty set is the simplex, then upper bounds can be found by using the techniques in Section V-A.

\section{E. Time-Delay Systems}

Time-delay systems is "before" another important Another important area of automatic control where optimization problems over polynomials finds application. For simplicity, let us consider the time-delay system

$$
\left\{\begin{array}{l}
\dot{x}(t)=A_{0} x(t)+A_{1} x(t-h) \forall t \geq 0 \\
x(t)=\phi(t) \forall t \in[-h, 0]
\end{array}\right.
$$

where $x \in \mathbb{R}^{n}$ is the state, $h \geq 0$ is the delay, and $\phi:[-h, 0] \rightarrow \mathbb{R}$ is the initial condition. The problem consists of establishing whether the system is exponentially stable, i.e., $x$ exponentially converges to the origin as $t \rightarrow \infty$ for all continuous functions $\phi$. 
As explained in [57], this problem can be addressed by defining the Lyapunov function

$$
\begin{aligned}
v(\phi)=\int_{-h}^{0}\left(\begin{array}{l}
\phi(0) \\
\phi(s)
\end{array}\right)^{\prime} & M(s)\left(\begin{array}{l}
\phi(0) \\
\phi(s)
\end{array}\right) d s \\
& +\int_{-h}^{0} \int_{-h}^{0} \phi(s)^{\prime} N(s, t) \phi(t) d s d t
\end{aligned}
$$

where $M:[-h, 0] \rightarrow \mathbb{S}^{2 n}$ and $N:[-h, 0] \times[-h, 0] \rightarrow \mathbb{R}^{n \times n}$ are matrix polynomials. Indeed, by expressing the Lie derivative of $v$ as

$$
\begin{aligned}
\dot{v}(\phi)=\int_{-h}^{0}\left(\begin{array}{c}
\phi(0) \\
\phi(-h) \\
\phi(s)
\end{array}\right) & D(s)\left(\begin{array}{c}
\phi(0) \\
\phi(-h) \\
\phi(s)
\end{array}\right) d s \\
& +\int_{-h}^{0} \int_{-h}^{0} \phi(s)^{\prime} E(s, t) \phi(t) d s d t
\end{aligned}
$$

one has that (71) is exponentially stable if there exist $M, N$ such that the matrix polynomials

$$
M(s)+\left(\begin{array}{cc}
T(s) & 0_{n \times n} \\
* & 0_{n \times n}
\end{array}\right),-D(s)+\left(\begin{array}{cc}
U(s) & 0_{n \times n} \\
* & 0_{n \times n}
\end{array}\right)
$$

are SOS, the symmetric matrix polynomials $T$ and $U$ satisfy

$$
\int_{-h}^{0} T(s) d s=0_{n \times n}, \int_{-h}^{0} U(s) d s=0_{2 n \times 2 n}
$$

and $N(s, t)$ and $-E(s, t)$ can be expressed as $s^{\{d\}^{\prime}} B t^{\{d\}}$ for some $d \in \mathbb{N}$ and $B \geq 0$. Hence, these conditions can be checked via LMI feasibility tests as described in Section III-A. See also [58] where this strategy is extended to the case of multiple delays and nonlinear systems.

\section{F. Genetic Regulatory Networks}

Optimization of polynomials finds application also in the novel area of systems biology. Indeed, let us consider the genetic network with SUM regulatory functions

$$
\left\{\begin{array}{l}
\dot{m}(t)=A(\theta) m(t)+b(\theta)+B(\theta) g(p(t)) \\
\dot{p}(t)=C(\theta) p(t)+D(\theta) m(t) \\
\theta \in[0,1]^{q}
\end{array}\right.
$$

where $m, p \in \mathbb{R}^{n}$ are the concentrations of mRNA and protein, $\theta$ is the uncertainty, $A, b, B, C, D$ are linear functions of suitable dimension, and $g: \mathbb{R}^{n} \rightarrow \mathbb{R}^{n}$ is defined by

$$
g_{i}(p)=\frac{p_{i}^{h}}{\beta^{h}+p_{i}^{h}}
$$

with $\beta \in \mathbb{R}$ and $h \in \mathbb{N}$. The problem consists of establishing whether the network is robustly stable, i.e., there exists a globally asymptotically stable equilibrium point for all admissible values of the uncertainty.

This problem can be addressed as proposed in [59]. Specifically, let $\left(m^{*}, p^{*}\right)^{\prime}$ be the (unknown) equilibrium point depending on $\theta$, and let us shift $\left(m^{*}, p^{*}\right)^{\prime}$ in the origin by defining $x=m-m^{*}$ and $y=p-p^{*}$. The network (76) can be rewritten as

$$
\left\{\begin{array}{l}
\dot{x}(t)=A(\theta) x(t)+B(\theta) h\left(y(t), p^{*}(\theta)\right) \\
\dot{y}(t)=C(\theta) y(t)+D(\theta) x(t)
\end{array}\right.
$$

for a suitable function $h$. Let $\mathcal{Z}(y, \theta) \subset \mathbb{R}^{n}$ be any set satisfying

$$
h\left(y, p^{*}(\theta)\right) \in \mathcal{Z}(y, \theta) \forall y \in \mathcal{Y}(\theta) \forall \theta \in[0,1]^{q}
$$

where

$$
\mathcal{Y}(\theta)=\left\{y \in \mathbb{R}^{n}: y_{i} \geq-p_{i}^{*}(\theta) \forall i=1, \ldots, n\right\} .
$$

The network (76) is robustly stable if there exists a polynomially parameter-dependent quadratic Lyapunov function $v(x, y, \theta)$ such that

$$
\left.\begin{array}{r}
v(x, y, \theta)>0 \\
\bar{v}(x, y, z, \theta)<0
\end{array}\right\} \forall\left(x^{\prime}, y^{\prime}\right)^{\prime} \in \mathbb{R}_{0}^{2 n} \forall z \in \mathcal{Z}(y, \theta) \forall \theta \in[0,1]^{q}
$$

where

$$
\bar{v}(x, y, z, \theta)=\frac{\partial v(x, y, \theta)}{\partial\left(x^{\prime}, y^{\prime}\right)^{\prime}}\left(\begin{array}{l}
A(\theta) x+B(\theta) z \\
D(\theta) x+C(\theta) y
\end{array}\right) .
$$

By describing the set $\mathcal{Z}(y, \theta)$ via polynomial inequalities and adopting the techniques in Sections IV-A and IV-B, this strategy provides a sufficient condition for robust stability of (76) via an LMI feasibility test. See [59] for details and examples.

\section{G. Game Theory}

Another interesting application is in game theory and has been proposed in [60]. Indeed, a typical problem in polynomial games with two players consists of solving

$$
\min _{\gamma, \mu} \gamma \text { s.t. }\left\{\begin{array}{l}
E_{\mu}[P(x, y)] \leq \gamma \forall x \in[-1,1] \\
\int_{-1}^{1} d \mu(y)=1
\end{array}\right.
$$

where $x, y \in[-1,1]$ are the actions of the players, $P: \mathbb{R} \times$ $\mathbb{R} \rightarrow \mathbb{R}$ is the payoff that assigns payments from Player 2 to Player $1, E_{\mu}$ denotes the expectation under the probability measure $\mu$, and $\gamma \in \mathbb{R}$ represents the gain of Player 1 if Player 2 plays the strategy $\mu$ obtained from the solution of this problem.

If $P$ is a polynomial expressed as

$$
P(x, y)=\sum_{i=0}^{n} \sum_{j=0}^{m} p_{i, j} x^{i} y^{j}
$$

for some $p_{i, j} \in \mathbb{R}$, this problem can be converted into

$$
\min _{\gamma, \mu} \gamma \text { s.t. }\left\{\begin{array}{l}
\gamma-\sum_{i=0}^{n} \sum_{j=0}^{m} p_{i j} x^{i} \mu_{j} \geq 0 \forall x \in[-1,1] \\
\sum_{j=0}^{m} \mu_{j} x^{j} \geq 0 \forall x \in \mathbb{R} \\
\sum_{j=0}^{m-2}\left(\mu_{j}-\mu_{j+2}\right) x^{j} \geq 0 \forall x \in \mathbb{R} \\
\mu_{0}=1
\end{array}\right.
$$


where $\mu_{0}, \ldots, \mu_{m}$ are the moments of the measure $\mu$, i.e.

$$
\mu_{j}=\int_{-1}^{1} y^{j} d \mu .
$$

The optimization problem (85) can be solved via an SDP by using the techniques in Sections III-A and IV-A based on SOS polynomials and Positivstellensatz. Indeed, since the polynomials in (85) are univariate, there is no gap between positive polynomials and SOS polynomials according to Theorem 2, moreover a necessary and sufficient condition via SOS polynomials for positivity over a closed interval is given by Theorem 7 . See [60] for details.

\section{CONCLUSION}

Solving optimization problems over polynomials is often required in various tasks of control systems. A useful way of addressing this issue consists of adopting LMI techniques, as they allow one to obtain bounds to the sought solution by solving convex optimization problems, and as the conservatism of these bounds can be decreased in general by suitably increasing the size of the problems. This survey has presented an overview of these LMI techniques and their main applications in control systems, in order to provide all readers with a concise useful reference.

\section{ACKNOWLEDGMENT}

The author would like to thank the Editors and the Reviewers for their valuable comments, and D. Henrion and C. Scherer for helpful discussions.

\section{REFERENCES}

[1] , D. Henrion and A. Garulli, Eds., Positive Polynomials in Control. New York: Springer, 2005.

[2] N. Z. Shor, "Class of global minimum bounds of polynomial functions (in russian)," Cybernetics, vol. 23, pp. 731-734, 1987.

[3] Y. Nesterov, "Squared functional systems and optimization problems," in High Performance Optimization, H. Frenk, K. Roos, T. Terlaky, and S. Shang, Eds. Norwell, MA: Kluwer, 2000.

[4] M. Choi, T. Lam, and B. Reznick, "Sums of squares of real polynomials," in Proc. Symp. Pure Math., 1995, pp. 103-126.

[5] G. Chesi, A. Tesi, A. Vicino, and R. Genesio, "On convexification of some minimum distance problems," in Proc. Eur. Control Conf., Karlsruhe, Germany, 1999, [CD ROM].

[6] P. A. Parrilo, "Structured Semidefinite Programs and Semialgebraic Geometry Methods in Robustness and Optimization," Ph.D. dissertation, California Inst. Technol., Pasadena, 2000.

[7] S. Boyd, L. El Ghaoui, E. Feron, and V. Balakrishnan, Linear Matrix Inequalities in System and Control Theory. Philadelphia, PA: SIAM, 1994.

[8] B. Reznick, "Extremal psd forms with few terms," Duke Math. J., vol. 45, no. 2, pp. 363-374, 1978.

[9] F. Wu and S. Prajna, "SOS-based solution approach to polynomial lpv system analysis and synthesis problems," Int. J. Control, vol. 78, no. 8, pp. 600-611, 2005.

[10] G. Chesi, A. Garulli, A. Tesi, and A. Vicino, "Robust stability for polytopic systems via polynomially parameter-dependent Lyapunov functions," in Proc. IEEE Conf. Decision Control, Maui, HI, 2003, pp. 4670-4675.

[11] C. W. J. Hol and C. W. Scherer, "Computing optimal fixed order $\mathcal{H}_{\infty}$-synthesis values by matrix sum of squares relaxations," in Proc. IEEE Conf. Decision Control, Paradise Island, Bahamas, 2004, pp. 3147-3153.
[12] G. Chesi, A. Garulli, A. Tesi, and A. Vicino, Homogeneous Polynomial Forms for Robustness Analysis of Uncertain Systems. New York: Springer, 2009.

[13] B. Reznick, "Some concrete aspects of Hilbert's 17th problem," Contemporary Math., vol. 253, pp. 251-272, 2000.

[14] G. Chesi, "On the gap between positive polynomials and SOS of polynomials," IEEE Trans. Autom. Control, vol. 52, no. 6, pp. 1066-1072, Jun. 2007.

[15] G. Hardy, J. E. Littlewood, and G. Pólya, Inequalities, 2nd ed. Cambridge, U.K.: Cambridge Univ. Press, 1988.

[16] J.-B. Lasserre, "A sum of squares approximation of nonnegative polynomials," SIAM J. Optim., vol. 16, pp. 761-765, 2006.

[17] G. Blekherman, "There are significantly more nonnegative polynomials than sums of squares," Israel J. Math., vol. 153, no. 1, pp. 355-380, 2006

[18] C. J. Hillar and J. Nie, "An elementary and constructive solution to Hilbert's 17th problem for matrices," in Proc. Amer. Math. Soc., 2008, vol. 136, pp. 73-76.

[19] J. Bochnak, M. Coste, and M.-F Roy, Real Algebraic Geometry. New York: Springer, 1998

[20] M. Laurent, "Sums of squares, moment matrices and optimization over polynomials," in Emerging Applications of Algebraic Geometry, M. Putinar and S. Sullivant, Eds. New York: Springer, 2009, vol. 149, pp. $157-270$.

[21] G. Stengle, "A nullstellensatz and a positivstellensatz in semialgebraic geometry," Math. Annu., vol. 207, pp. 87-97, 1974.

[22] K. Schmuedgen, "The K-moment problem for compact semi-algebraic sets," Mathematische Annalen, vol. 289, pp. 203-206, 1991.

[23] M. Putinar, "Positive polynomials on compact semi-algebraic sets," Ind. Univ. Math., vol. 42, pp. 969-984, 1993.

[24] P. A. Parrilo, "Semidefinite programming relaxations for semialgebraic problems," Math. Programming Ser. B, vol. 96, no. 2, pp. 293-320, 2003.

[25] C. W. Scherer and C. W. J. Hol, "Matrix sum-of-squares relaxations for robust semi-definite programs," Math. Programming Ser. B, vol. 107, no. 1-2, pp. 189-211, 2006.

[26] M. Kojima, Sums of Squares Relaxations of Polynomial Semidefinite Programs Tokyo Inst. Technol., Tokyo, Japan, Tech. Rep., 2003.

[27] C. W. J. Hol and C. W. Scherer, "Fixed order $\mathcal{H}_{\infty}$-synthesis: Computing optimal values by robust performance analysis," in Proc. Amer. Control Conf., Boston, MA, 2004, pp. 3285-3290.

[28] D. Henrion and J.-B. Lasserre, "Convergent relaxations of polynomial matrix inequalities and static output feedback," IEEE Trans. Autom. Control, vol. 51, no. 2, pp. 192-202, Feb. 2006.

[29] J.-B. Lasserre, "Global optimization with polynomials and the problem of moments," SIAM J. Optim., vol. 11, no. 3, pp. 796-817, 2001.

[30] C. W. Scherer, "Relaxations for robust linear matrix inequality problems with verifications for exactness," SIAM J. Matrix Anal. Appl, vol. 27, no. 1, pp. 365-395, 2005.

[31] V. Powers and B. Reznick, "A new bound for polya's theorem with applications to polynomials positive on polyhedra," J. Pure Appl. Algebra, vol. 164, pp. 221-229, 2001.

[32] G. Chesi, A. Garulli, A. Tesi, and A. Vicino, "Solving quadratic distance problems: an LMI-based approach," IEEE Trans. Autom. Control, vol. 48, no. 2, pp. 200-212, Feb. 2003.

[33] M. C. de Oliveira, J. Bernussou, and J. C. Geromel, "A new discrete-time robust stability condition," Syst. Control Lett., vol. 37, pp. 261-265, 1999.

[34] Y. Oishi, "A matrix-dilation approach to robust semidefinite programming and its error bound," in Proc. Amer. Control Conf., Minneapolis, MI, 2006, pp. 123-129.

[35] M. Sato and D. Peaucelle, "Comparison between SOS approach and slack variable approach for non-negativity check of polynomial functions: Multiple variable case," in Proc. Eur. Control Conf., Kos, Greece, 2007, pp. 3016-3025.

[36] D. Henrion and J.-B. Lasserre, "GloptiPoly: Global optimization over polynomials with Matlab and SeDuMi," in Proc. IEEE Conf. Decision Control, Las Vegas, NV, 2002, pp. 747-752.

[37] S. Prajna, A. Papachristodoulou, P. Seiler, and P. A. Parrilo, SOSTOOLS: Sum of Squares Optimization Toolbox for MATLAB. Natick, MA: The Mathworks Inc., 2004

[38] J. Lofberg, "YALMIP: a toolbox for modeling and optimization in MATLAB," in Proc. CACSD Conf., Taipei, Taiwan, 2004, pp. 284-289. 
[39] H. K. Khalil, Nonlinear Systems. Englewood Cliffs, NJ: Prentice Hall, 2001.

[40] A. Papachristodoulou and S. Prajna, "On the construction of Lyapunov functions using the sum of squares decomposition," in Proc. IEEE Conf. Decision Control, Las Vegas, NV, 2002, pp. 3482-3487.

[41] B. Tibken, "Estimation of the domain of attraction for polynomial systems via LMI's," in Proc. IEEE Conf. Decision Control, Sydney, Australia, 2000, pp. 3860-3864.

[42] W. Tan and A. Packard, "Stability region analysis using polynomial and composite polynomial Lyapunov functions and sum-of-squares programming," IEEE Trans. Autom. Control, vol. 53, no. 2, pp. 565-571, Jan. 2008

[43] G. Chesi, "Estimating the domain of attraction for non-polynomial systems via LMI optimizations," Automatica, vol. 45, no. 6, pp. $1536-1541,2009$.

[44] A. Garulli, A. Masi, and A. Vicino, "Relationships among different SOS-based relaxations for quadratic distance problems," in Proc. IFAC Symp. Robust Control Design, Haifa, Israel, 2009, pp. 66-71.

[45] M. Peet, "Exponentially stable nonlinear systems have polynomial Lyapunov functions on bounded regions," IEEE Trans. Autom. Control, vol. 54, no. 5, pp. 979-987, May 2009.

[46] R. C. L. F. Oliveira and P. L. D. Peres, "Parameter-dependent LMIs in robust analysis: Characterization of homogeneous polynomially parameter-dependent solutions via LMI relaxations," IEEE Trans. Autom. Control, vol. 52, no. 7, pp. 1334-1340, Jul. 2007.

[47] P.-A. Bliman, "A convex approach to robust stability for linear systems with uncertain scalar parameters," SIAM J. Control Optim., vol. 42, no. 6, pp. 2016-2042, 2004.

[48] M. Sato, "Robust stability/performance analysis for polytopic systems via multiple slack variable approach," in Proc. 17th IFAC World Congress, Seoul, Korea, 2008, pp. 11391-11396.

[49] Y. Ebihara, Y. Onishi, and T. Hagiwara, "Robust performance analysis of uncertain LTI systems: Dual LMI approach and verifications for exactness," IEEE Trans. Autom. Control, vol. 54, no. 5, pp. 938-951, May 2009.

[50] D. Henrion, D. Arzelier, D. Peaucelle, and J.-B. Lasserre, "On parameter-dependent Lyapunov functions for robust stability of linear systems," in Proc. IEEE Conf. Decision Control, Paradise Island, Bahamas, 2004, pp. 887-892.

[51] R. W. Brockett, "Lie algebra and lie groups in control theory," in Geometric Methods in Systems Theory, D. Q. Mayne and R. W. Brockett, Eds. Dordrecht, The Netherlands: Springer, 1973, pp. 43-82.

[52] A. L. Zelentsovsky, "Nonquadratic Lyapunov functions for robust stability analysis of linear uncertain systems," IEEE Trans. Autom. Control, vol. 39, no. 1, pp. 135-138, Jan. 1994.

[53] G. Chesi, A. Garulli, A. Tesi, and A. Vicino, "Homogeneous Lyapunov functions for systems with structured uncertainties," Automatica, vol. 39, no. 6, pp. 1027-1035, 2003.
[54] F. Blanchini and S. Miani, "A universal class of smooth functions for robust control," IEEE Trans. Autom. Control, vol. 44, no. 3, pp. 641-647, Mar. 1999.

[55] C. W. Scherer, "LMI relaxations in robust control," Eur. J. Control, vol. 12, no. 1, pp. 3-29, 2006.

[56] J. Lavaei and A. G. Aghdam, "Robust stability of LTI systems over semi-algebraic sets using sum-of-squares matrix polynomials," IEEE Trans. Autom. Control, vol. 53, no. 1, pp. 417-423, Feb. 2008.

[57] M. Peet, A. Papachristodoulou, and S. Lall, "Positive forms and stability of linear time-delay systems," in Proc. IEEE Conf. Decision Control, San Diego, CA, 2006, pp. 187-192.

[58] M. Peet, A. Papachristodoulou, and S. Lall, "Analysis of polynomial systems with time delays via the sum of squares decomposition," IEEE Trans. Autom. Control, vol. 54, no. 5, pp. 1058-1064, May 2009.

[59] G. Chesi and Y. S. Hung, "Stability analysis of uncertain genetic SUM regulatory networks," Automatica, vol. 44, no. 9, pp. 2298-2305, 2008.

[60] P. A. Parrilo, "Polynomial games and sum of squares optimization," in Proc. IEEE Conf. Decision Control, San Diego, CA, 2006, pp. $2855-2860$.

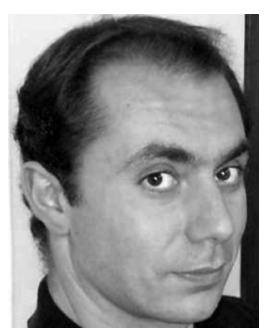

Graziano Chesi (SM'06) received the Laurea degree in information engineering from the University of Florence, Florence, Italy, in 1997 and the Ph.D. degree in systems engineering from the University of Bologna, Bologna, Italy, in 2001.

He was with the Department of Information Engineering, University of Siena, Siena, Italy, from 2000 to 2006 and then joined the Department of Electrical and Electronic Engineering, University of Hong Kong, 2006-present. He was a Visiting Scientist at the Department of Engineering, University of Cambridge, Cambridge, U.K., 1999 to 2000, and at the Department of Information Physics and Computing, University of Tokyo, Tokyo, Japan, 2001 to 2004. Presently, he serves as Associate Editor of Automatica, BMC Research Notes, and the European Journal of Control. He is author of Homogeneous Polynomial Forms for Robustness Analysis of Uncertain Systems (Springer, 2009) and Editor of Visual Servoing via Advanced Numerical Methods (Springer, 2010). $\mathrm{He}$ is first author in more than 100 technical publications.

Dr. Chesi received the Best Student Award of the Faculty of Engineering, University of Florence, Florence, Italy, in 1997. He was Associate Editor of the IEEE TRANSACTIONS ON AUtOMATIC CONTROL (2005-2009) and Guest Editor of the Special Issue on Positive Polynomials in Control of the IEEE Transactions ON Automatic CONTROL (2009). He is the Founder and Chair of the Technical Committee on Systems with Uncertainty of the IEEE Control Systems Society. 